\title{
Neue Studie über ein altes Therapieprinzip
}

\section{Ob man bei Patienten mit akuter dekompensierter Herzinsuffizienz Schleifendiuretika als Bolus oder als Infusion gibt, ob man sie hoch oder niedrig dosiert, hat für den Ausgang der Erkrankung keine wesentliche Bedeutung.}

— Die akute dekompensierte Herzinsuffizienz gehört mit zu den häufigsten Gründen für eine stationäre Krankenhausbehandlung. Schleifendiuretika sind seit Jahrzehnten für die Akuttherapie dieser Patienten unverzichtbar. Trotz der langen Erfahrung mit diesen Substanzen sind prospektive und kontrollierte Studien bisher kaum durchgeführt worden.

Die gegenwärtigen Leitlinien basieren überwiegend auf Expertenmeinungen. In der DOSE-Studie wurden 308 Patienten mit akuter dekompensierter Herzinsuffizienz in prospektiver und doppelblinder Weise mit Furosemid, appliziert entweder alle zwölf Stunden als Bolus oder als kontinuierliche Infusion in entweder niedriger Dosis (ähnlich der Dosis, die der Patient zuvor oral eingenommen hatte) oder hoher Dosis (2,5 x die vorherige orale Dosis), behandelt. Endpunkte waren die allgemeine Beurteilung der Symptome durch den Patienten anhand einer visuellen Analogskala und die Änderung des Serumkreatinins nach $72 \mathrm{~h}$.

Für keines der vier Therapieprinzipien ließ sich ein signifikanter Vorteil erkennen. Auch die Serumkreatininwerte änderten sich in den vier Gruppen nicht wesentlich. Die Hochdosis-Strategie führte zu einer stärkeren Diurese und wies Vorteile bei einigen sekundären Ergebnissen auf, ging allerdings auch mit einer vorübergehenden Verschlechterung der Nierenfunktion einher.

Kardiomegalie bei Herzinsuffizienz mit rückläufigem Pleuraerguss links unter diuretischer Therapie.

\section{Kommentar}

Das Ergebnis der Studie reißt den Kliniker nicht gerade vom Stuhl, aber nun haben wir die wissenschaftliche Evidenz, dass es nicht so entscheidend ist, ob Schleifendiuretika im Bolus oder als Infusion, niedrig oder hoch dosiert gegeben werden. Unabhängig von dem bescheidenen klinischen Ergebnis weist die Studie ein interessantes Detail auf: Sie wurde vollständig vom amerikanischen National Heart, Lung and Blood Institute finanziert und nicht von einer Pharmafirma. Ob ein Medikament, das seit Jahrzehnten zum therapeutischen Standardrepertoire gehört, heute immer noch seine Berechtigung hat und in welcher Weise es gegeben wird, interessiert die pharmazeutische Industrie nicht mehr. Manche Substanzen profitieren bis heute von der "Gnade der frühen Geburt", da bei ihrer Einführung wesentlich niedrigere Effizienz- und Sicherheitsstandards gültig waren, als dies heute der Fall ist.

H. S. FÜESSL ,

- G. M. Feldkamp et al.

(Duke Clinical Research Institute, 2400 Pratt St., Rm 0311 Terrace Level, Durham, NC 27705): Diuretic strategies in patients with acute decompensated heart failure. New Engl. J Med. 364 (2011) 9, 797-805

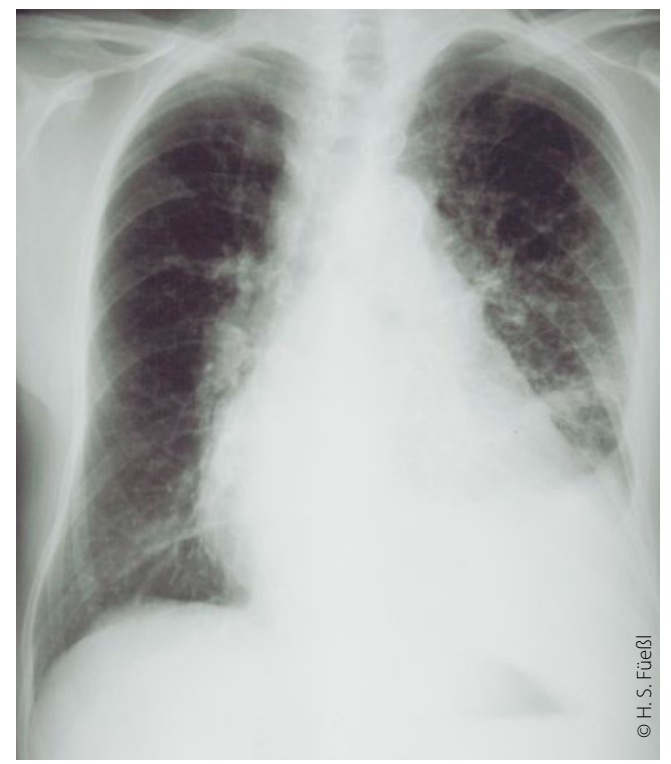

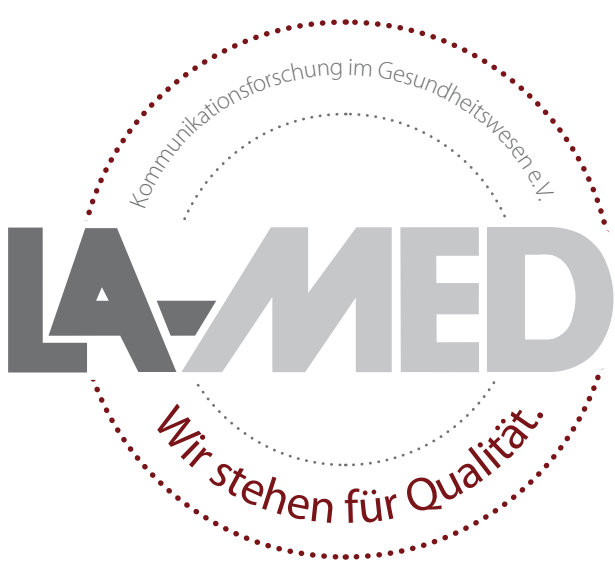

Sie lesen
Qualität

Damit das auch so bleibt, befragen wir Sie in Kooperation mit tns infratest in den nächsten Wochen.

Ihr Urteil ist uns wichtig. Bitte nehmen Sie teil! 